\title{
The risk of consuming MTGase-restructured steaks like intact steaks
}

\author{
Denny Zheng ${ }^{1}$, Bobby Sidhu ${ }^{2}$, Ken Keilbart ${ }^{3}$, \& Lorraine McIntyre ${ }^{4}$ \\ 1. Lead author, B. Tech student, School of Health Sciences, British Columbia Institute of Technology, 3700 Willingdon Ave Burnaby, BC V5G 3H2 \\ 2. Supervisor, School of Health Sciences, British Columbia Institute of Technology, 3700 Willingdon Ave Burnaby, BC V5G 3H2 \\ 3. Contributor, Food Technology Laboratory, School of Health Sciences, British Columbia Institute of Technology, 3700 Willingdon Ave Burnaby, BC V5G 3H2 \\ 4. Contributor, British Columbia Centre for Disease Control, 655 West 12th Avenue Vancouver, BC V5Z 4R4
}

\begin{abstract}
:
Introduction: The use of transglutaminase to restructure loose pieces of meat into a fully intact piece of steak has been a concern for the public because of the potential internalization of contaminated surfaces into the aseptic center. The aim of this study was to examine if restructured steaks are safe to consume when cooked to medium rare, a common option with whole cut steaks
\end{abstract}

Methods: Strips of beef were inoculated with E.coli to induce surface contamination. Steaks were restructured with transglutaminase and the altered meat. These steaks were then cooked alongside fully intact whole-cut steak samples. Each sample was then churned in a stomacher, and the resulting solution was used to detect for potential E.coli bacteria. Samples were then enriched and finally placed into the Hygiena Micro-snap Rapid Coliform and E.coli detection test to look for the presence of E.coli.

Results: The Hygiena system showed that all transglutaminase restructured steaks possessed detectable levels of E.coli even after cooking to 55 degrees Celsius. On the other hand, no whole-cut steaks had traces of E.coli even when cooked to this same temperature.

Conclusion: The results demonstrated that there is a substantial risk with restructured steaks and they should not be consumed undercooked. As well, proper labelling and guidelines should be developed to enable consumers to be better equipped in making decisions to consume properly consumed altered steaks.

Keywords: Transglutaminase, meat glue, steaks, food safety, Escherichia coli

\section{Introduction}

One of the most important aspects of public health that needs to be continuously monitored is food safety. However with a constantly increasing number of new food items and processing methods, the knowledge and skills available to manage risk is at an arms race with the creation of new products. Federal and provincial bodies are continuously assessing these different foods, and frequently formulating new guidelines to assist regulators (Catford et al., 2013).
An example of such products that has come into the public's awareness is food that is created through the use of meat glue, whose proper name is transglutaminase. As Bottemiller (2012) pointed out, there is concern over whether restructured steaks have higher associated risks and whether consumers should be informed of these risks. Steaks are one type of meat that are generally considered safe to eat undercooked because the center is aseptic, and 
surface searing during cooking would inactivate all pathogens (Bottemiller, 2012). For this reason they are known as intact meats. Transglutaminaserestructured steaks could internalize the surfaces of individual pieces, and the concern is focused on the lack of an aseptic center like whole-cut steaks.

However Echeverry et al (as cited in Chancey, 2010) has stated the internal environment may not allow for growth of microbes, and therefore perhaps securing safety of the food. The purpose of this study was to look at whether there is there an increased risk with consuming medium rare transglutaminaserestructured steaks compared to medium rare wholecut steaks?

The hypotheses designed to be tested were: $\mathrm{H}_{0}$ : There is no association between the presence of E.coli in medium-rare transglutaminase restructured steaks compared to medium-rare whole-cut steaks $\mathrm{H}_{\mathrm{a}}$ : There is an association between the presence of E.coli in medium-rare transglutaminase restructured steaks compared to medium-rare whole-cut steaks.

\section{Literature Review}

\section{Transglutaminase: A food processing agent}

Food modification occurs in all types of food that we consume on a daily basis. There are a variety of techniques available to improve foods in either quality or quantity. The use of Transglutaminase is one such technique that has been used in the food industry for decades, and is intended for creating stronger structural integrity to improve the quality of the final product (Motoki \& Seguro, 1998).

Transglutaminase (TG) is commonly found as a microbial enzyme but has a counterpart in humans, commonly found in human body fluid and tissue cells (Gerrard \& Cottam, 2012). Its role in the human body is involved in blood clotting and wound healing. When it was discovered in the 1980s that TG had the potential to being used in increasing overall bonds within foods there was interest in utilizing it at a larger scale (Motoki \& Seguro, 1998). However, the cost of isolating TG from animal sources made it difficult to be accepted as a binding agent (Gerrard \& Cottam, 2012). This changed when researchers were able to isolate bacterial TG from the bacteria Streptoverticillium mobaraense which then allowed increased usage of TG (Motoki \& Seguro, 1998). This bacterial form of transglutaminase is commonly referred to as microbial transglutaminase (MTGase).

\section{Transglutaminase: A threat to food safety?}

After more than three decades of use, MTGase is now incorporated in the preparation of many types of food. It is used with dairy products such as yogurts, cheeses, and ice cream (Motoki \& Seguro, 1998).

Breads can also be incorporated with transglutaminase to produce a more favorable product (De Jong \& Koppelman, 2002). Finally, a significant portion of transglutaminase use is in the meat industry. According to De Jong \& Koppelman (2002), the use of MTGase in meat products is good for helping to create bonds between muscle fibers, and creating new crosslinks between pieces of meats attached. This allowed for food processors and food establishments to use the enzyme to create new pieces of meat from smaller individual portions.

The concern addressed in this study is not based on any toxicological concerns with the use of MTGase. The FDA has granted MTGase with Generally Recognized as Safe (GRAS) status since 1998, which was given after a review of relevant studies that looked at the toxicity of MTGase (FDA, 2001). Instead, the concern over these food products is focused on the risk of disease or illnesses that can be attributable to the unnatural physical changes in the final meat product. Specifically, the main cause of concern is whether there is a risk with consuming restructured meats that do not reach an internal temperature of 71 degrees Celsius. It is therefore important for public health officials to be aware of such new products which may jeopardize food safety in order to limit illnesses from occurring.

\section{Escherichia coli and disease}

Pathogens must be found on the surface of food prior to any foodborne illnesses occurring, and this is no exception for restructured foods. With restructured steaks, the main organism of concern would be E.coli O157:H7. E.coli O157:H7 belongs to the group of enterohemorrhagic E.coli and causes bloody diarrhea, abdominal pain, and vomiting in people who 
consume foods contaminated with it (Centers for Disease Control and Prevention (CDC), 2012). This type of E.coli is naturally found in all types of animals including cattle, goats, sheep, deer, elk and many others. Food is usually contaminated with this pathogen through any one of the following means: consuming improperly cooked beef, contaminated fruits, uncooked vegetables, raw milk, untreated drinking water, or not properly washing hands after touching meats (Public Health Agency of Canada (PHAC), 2013; Huang \& Sheen, 2011).

Symptoms will begin to show up on average around three to four days, but could occur up to ten days later (PHAC, 2013). For most people the infection will end when these symptoms end but for other individuals they can develop sequelae, or postinfection conditions. One particularly devastating sequelae is known as hemolytic uremic syndrome, which involves failure of the kidneys (CDC, 2012). These conditions generally occur in the elderly, youths, or the immune-compromised inflicted with an E.coli O157:H7 infection. According to Catford et al. (2013), it only takes 100-200 E.coli cells to cause an infection, making it a very potent pathogen. Due to the severity and commonality of E.coli with foodborne illnesses with meat, it was the chosen organism to be tested for in this study.

\section{Effect of foodborne illnesses on health and economics}

Another reason for preventing foodborne illnesses is due to the significant costs on society. In Canada, the current estimate of the incidence rate of GI illnesses is 1.3 cases per 1 person year (Vrbova, Johnson, Whitfield, \& Middleton, 2012). An equally shocking statistic is that a person has a $72 \%$ chance of developing a GI illness in any year. Economic costs have been estimated to be about $\$ 113$ per capita or slightly over \$1000 per case that develops. Another estimate tells us that there is an astonishing 4.0 million cases of foodborne illnesses in Canada per year (Thomas et al., 2013). All together, the harm of foodborne illnesses, and the cost of it on our economy provide sufficient grounds for continuing to push our understanding of common causes and preventing their occurrences.

\section{Internally contaminated steaks and diseases}

While it can be appreciated that there is a real potential of restructured steaks causing foodborne illnesses, actual experimental studies examining the risks of transglutaminase-dependent steaks have not been documented. However the effect that internalized pathogens have on health has been explored in the past through looking at other food processing methods that can also introduce pathogens internally, such as mechanical tenderization.

Mechanical tenderizing is a processing method that disrupts the rough structure of meat so that it becomes more appealing to the consumer (Huang \& Sheen, 2011). The treatment is performed by using a machine that is equipped with a series of blades or needles and will pierce through a portion of meat. This interaction will physically break apart the structure of the meat. Mechanical tenderization can introduce pathogens through the penetration of the meat by needles past the surface into the internal environment (Huang \& Sheen, 2011).

This method of processing meat acts as a good model for transglutaminase restructured steaks because:

1) Both practices are commonly implemented in the final retail or consumption stage of meat distribution and

2) Both practices can introduce contamination into the center of the meat.

While the processes between tenderized and restructured steaks are not comparable, the effects of internalizing pathogens with mechanical tenderized steaks can be extrapolated to restructured steaks.

One aspect that can be observed using mechanically tenderized meats as a model is the contribution of internally contaminated meats in outbreaks. Catford et al. (2013) revealed that there were a total of 18 E.coli O157:H7 cases where the investigations led to mechanical tenderizers as the cause of contamination. As well, in recent years larger outbreaks have been identified, with 4 in the United States and 1 in Canada, all of which had mechanical tenderizers implicated as the cause.

Both MTGase-restructured steaks and mechanical tenderized steaks belong to a group of meat known as non-intact meats. Catford et al. (2013) reveals that 
compared to intact meats, mechanically tenderized beef (MTB) are 5 times more dangerous to consume. However, truly non-intact meat like ground beef is 1500 and 7300 times more dangerous compared to MTBs and intact steaks respectively. Therefore it can be reasoned that MTGase-restructured steaks may have the same risk associated with foodborne illnesses as the other non-intact meats.

\section{Legislation on using reformed meat}

Current advice to mitigate problems with consumption of MTBs, which can be applied to MTGase-restructured steaks, only looks at providing guidelines for the consumer. The Canadian Public Health Association (CPHA) (2012) advises that consumers forego eating rare or medium-rare steaks, and instead have steaks cooked to an internal temperature of 74 degrees Celsius, correlating with a medium steak. This group also outlines the general PHAC and Health Canada's food safety practices for using non-intact meats: washing hands before and after cooking, keeping knives and counters clean, keeping raw meats separate from other foods, and keeping leftovers refrigerated quickly. While these are good general practices for preventing contamination, they are not specific enough for nonintact steaks served in food establishments. Thus for MTGase-restructured steaks, there needs to be better knowledge on this subject for restaurants operators and consumers. A significant issue with putting out guidelines for non-intact meats, as pointed out by Catford et al. (2013), is that there lacks consensus on appropriate temperature and other information on proper handling of these foods. Therefore more information needs to be collected before any guidelines can be set up.

\section{Methodology Preparatory Work}

Lauryl tryptose broths (LSB) (Difco, 0241-17) were prepared before the experiment as media for bacteria cultures, along with $9 \mathrm{~mL}$ tubes of $0.1 \%$ peptone water to be used for dilutions. These solutions were autoclaved and placed into a 4 degrees Celsius fridge until needed.

\section{Meat Acquisition}

Sirloin steaks were acquired from a local grocer for creating samples. Steaks were frozen until the day prior to experimentation, in which they were then allowed to thaw in the fridge. Steaks were divided into two groups depending on what type of samples they were used for: Whole-cut and Restructuredsteaks.

\section{E.coli culture preparation}

A purified plate culture of E.coli was retrieved and a sample was used to inoculate $9 \mathrm{~mL}$ of LSB media. The E.coli culture was supplied by the BCIT food technology laboratory. These inoculated LSB tubes were then put into a 35 degrees Celsius incubator for 12-18 hours.

\section{Inoculation of meat for restructured steaks}

Tubes of E.coli culture were taken out after the appropriate incubating time. The number of cells after this incubation was approximately $10^{9} \mathrm{cfu} / \mathrm{ml}$ (Ken Keilbart, personal communication, 2013). Serial dilutions were conducted to acquire the appropriate level of E.coli $\left(10^{5} \mathrm{cfu} / \mathrm{ml}\right)$ for inoculating meats. Chancey (2010) demonstrated that inoculating surfaces of meat with E.coli culture will result in a $1 / 100$ dilution of bacterial count on surfaces of meat. Steaks were cut up into thin pieces of meat ranging from 10-15 grams in mass. Each piece of meat then received $10 \mathrm{~mL}$ of $10^{5} \mathrm{cfu} / \mathrm{ml}$ E.coli culture across the surfaces of the meat. Each piece was then allowed to sit for 15 seconds for attachment of bacteria onto the surface (Chancey, 2010). A final concentration of $10^{3} \mathrm{cfu} / \mathrm{mL}$ of E.coli on surfaces of meat was used because previous research had demonstrated that this concentration was found on meat surfaces

(Elizaquivel, P., Gabaldon, J.A., \& Aznar, R., 2011).

\section{Creating Restructured Steaks}

After air drying, pieces of meat were then transferred to a pan to allow for application of transglutaminase. Moo Glue RM Transglutaminase (Modernist Pantry, ID: 1201-50) was evenly sprinkled on top of the strips of meat. Slices of meat were then put on top of each other to coordinate for a structure that resembles the desired structure of the final product (Burton, 2012). This combined meat was then transferred to plastic wrap for sealing the meats in order for the 
enzyme to carry out its activity. The roll of meat was then placed into a 4 degrees refrigerator to allow for meat binding overnight.

\section{Preparation of Steaks for Cooking:}

Both restructured meat and whole-cut steaks were taken out of the fridge. A clean knife was used to create medallion shaped steaks from the pre-formed roll of restructured meat. Whole-cut steaks were also shaped with a clean knife into similarly sized medallions. All medallions were roughly 1 inch thick, and on average had a diameter of 3-5 centimeters.

\section{Cooking of Sample Meat}

A frying pan was heated and a piece of restructured steak samples and a piece of whole-cut samples were cooked on it. A bi-metallic thermometer was inserted into each sample to monitor the internal temperature until it reached the desired temperature. Steaks were cooked until they reached an internal temperature of $135{ }^{\circ} \mathrm{F}$ or $55^{\circ} \mathrm{C}$ (Foodnetwork, nd; Rattray, nd.). Steaks were removed from the pan and placed on to separate plates for cooling. Thermometers were sterilized with boiling water in between uses to prevent cross contamination of samples.

\section{Preparation of Samples for Detection of E.coli}

Steaks were measured out on a scale and then placed into stomacher bag with $0.1 \%$ peptone water to create a 1 in 10 dilution heterogenous solution. Note that the first 7 samples of the experiment were processed differently. Only 1 gram samples were taken and these were mixed with $9 \mathrm{ml}$ of $0.1 \%$ peptone water to get a 1 in 10 dilution. Subsequent samples used the entire sample to process for E.coli detection.

The stomacher bag was then placed into a stomacher (Seward Stomacher 400) and ran through a high speed cycle for 2 minutes. $1 \mathrm{~mL}$ of the meat solution was removed from the stomacher bag and placed into an enrichment tube from the Hygiena Micro-snap Rapid Coliform and E.coli Detection test (Hygiena, cat: MS-EB-CE-100). Then the enrichment broth was released into the tube, and this enrichment tube was incubated in a 37 degrees Celsius incubator for 8 hours.

\section{Detection of E.coli with Detection Device:}

The Hygiena Micro-snap Rapid Coliform and E.coli detection tube was allowed to sit in room temperature for a minimum of 10 minute. The Hygiena SystemSURE II detection device was turned on so that it could equilibrate at room temperature (Hygiena, 2013a). The enrichment tube was then removed from the 37 degrees Celsius incubator. 0.1 $\mathrm{mL}$ of the enrichment tube solution was extracted and placed in a detection tube from the Hygiena Microsnap Rapid Coliform and E.coli detection series (Hygiena, cat: MS-EB-CE-100) (Hygiena, 2013a). The detection broth was then released into the content of the detection tube. The detection tube was then incubated for 10 minutes in the 37 degrees Celsius incubator. Finally, the detection tube was then placed into the Hygiena SystemSure II detection device after which a reading in relative light units (RLU) was acquired to determine the presence of E.coli in the solution. Presence was determined if RLU readings were equal or greater than 2 .

\section{Statistical Analysis Type of data}

The data collected from this study were entirely nominal dichotomous data. The study was to see if there was E.coli present or absent in the steaks after cooking.

\section{Descriptive statistics}

When dealing with nominal data, the data collected were broken up into its corresponding group (e.g. Presence or absence of E.coli). Doing this created a contingency table that showed the proportion of samples under each group.

\section{Inferential statistics:}

The best inferential statistic approach for the data gathered from a study such as this one was a chisquare test. This allows us to look for an association between the independent and dependent variables.

\section{Statistical packages}

The main tools used to carry out the descriptive and inferential statistical analyses were Microsoft Excel (Excel, 2010) and NCSS respectively (Hintze, 2013). 


\section{Results}

Through detection with the Hygiena micro-snap rapid coliform detection test, the number of steaks contaminated with E.coli and those that were not was determined.

A summary of the nominal data can be found in table 1. All 13 restructured steaks samples had the presence of E.coli detected. The whole-cut steaks had no samples found with E.coli after cooking.

Table 1: Proportion of restructured steaks and whole-cut steaks that were detected with E.coli or were not detected with E.coli

\begin{tabular}{|c|c|c|c|}
\hline Type of Steak & $\begin{array}{c}\text { Number } \\
\text { of } \\
\text { Samples } \\
\text { with } \\
\text { presence } \\
\text { of E.coli }\end{array}$ & $\begin{array}{c}\text { Number } \\
\text { of } \\
\text { Samples } \\
\text { with no } \\
\text { E.coli } \\
\text { presence }\end{array}$ & Total \\
\hline $\begin{array}{c}\text { Transglutamin } \\
\text { ase } \\
\begin{array}{c}\text { Restructured } \\
\text { steaks }\end{array}\end{array}$ & 13 & 0 & 13 \\
\hline $\begin{array}{c}\text { Whole-cut } \\
\text { steaks }\end{array}$ & 0 & 13 & 13 \\
\hline & & & 26 \\
\hline
\end{tabular}

This same data was put into NCSS 9 for an analysis using the chi-square test. A review of the print out from NCSS 9 confirmed that information was inputted correctly. This involved looking at the number of counts and whether it actually correlated with the number of samples that were tested. The program also generated its own contingency tables which the display of data in table 1 . The most crucial piece of information to this study was found in the different tests that were performed to look at a possible association between the independent and dependent variable. The preferred test for chi-square was the Pearson's Chi-Square test (Heacock, personal communication, 2013). In order to determine the significance of the Pearson's Chi-Square test results, it was important to analyze the p-value calculated. Indeed, the p-value reported in the printout, 0.00000 was lower than the baseline of 0.05 , which supported the significance of the results. As well, because the p-value is very low, the results supported an association between the presence of E.coli in medium-rare cooked restructured steaks compared to medium-rare cooked whole-cut steaks. Therefore, NCSS 9 formally supported rejecting the $\mathrm{H}_{\mathrm{o}}$ when the p-value is compared with $\alpha=0.05$.

Given that the p-value, 0.00000 , is much smaller than a stricter alpha value of 0.01 , there is no concern of a potential alpha error with rejecting the $\mathrm{H}_{\mathrm{o}}$.

\section{Discussion}

Techniques and processes available and utilized in our food supply is always being developed, and each can contribute to the level of complexity in processing that food undergoes prior to reaching the consumer. From a public health perspective, the incorporation of new processes is not a concern unless they contribute to risks associated with consuming the product. This is the concern with new techniques, as an added level of complexity in processing will make it harder for food security to be safeguarded (Newell et al., 2010).

With that in mind, the goal of this study was to enhance understanding of one such processing aid/technique involving the use of a binder to restructure pieces of meat. Transglutaminase is a known agent that improves structures of food by enzymatically inducing more interconnected bonds between proteins. The focus here was on the possible increase in inherent risks in consuming meats processed with transglutaminase. The results acquired from the described experiment demonstrated that restructured steaks were not sterilized after being heat treated to an internal temperature of 55 degree Celsius (common internal temperatures for mediumrare steaks). In comparison, intact steaks were fully sterilized after being exposed to the same heat treatment, which supports the common conception that surface cooking is sufficient in inactivating micro-organisms on the surface of steaks. Putting this together, we saw that the potential for the survival of internalized bacteria in re-constructed steaks can be a legitimate concern, and it must not be consumed in the same manner as whole-cut steaks.

Sufficient evidence has been analyzed in the past to support the claim that transglutaminase does not 
directly cause any health effects (FDA, 2001). However, the capability of a food items to harbor pathogen growth or offer protection from heat treatment is a serious issue that cannot go unaddressed. As mentioned by the CDC (2012) E.coli, which is the major pathogen associated with raw beef, can cause a very uncomfortable and potentially deadly disease within humans. As such, improper consumption of these meats can lead to unnecessary ailments in individuals which could otherwise be avoided with proper cooking. As well, Vrbova et al. (2012) and Thomas et al. (2013) both forewarned of the costs on the health care system and economic system from direct and indirect consequences of foodborne illnesses. This unnecessary use of available resources could otherwise be put into dealing with less preventable issues.

Previously, mechanically tenderized beefs (MTBs) were examined as a model for internalized pathogens and the potential of contributing to foodborne illnesses. The findings in this study also reinforced the similarities between the two groups of products. Both these processed products can be purchased by the public or consumed in restaurants. As well finals products from both processes can potentially harbor enough pathogens on the internal structure to cause illnesses when they are not inactivated (Huang \& Sheen, 2013). Therefore MTGase restructured meats and mechanically tenderized meats should be looked at together when considering measures to control their risks. The same should be done with other similar processing methods that can affect the integrity of meat. Any attempts at educating the public and processors of the potential harm from these resulting food products should also be done in a concerted effort.

Appropriate actions need to be carried out to help control the risk of consuming this type of meat. Processors and consumers both need to be included or advised of the risks with restructured steaks in order to be able to safeguard food security. Firstly, processors should be given the onus to alert the public of any products containing or created with the use of transglutaminase. If preventative action is to be successful, then it is important that this transparency with the type of food being supplied to the market needs to be established. Hence, a good start would be to use labelling or advisories as a way to help processors convey this information to consumers. Consumers themselves will need to be supplied with the appropriate information on how to handle restructured steaks either when consuming the steaks in restaurants, or preparing the steaks themselves. The key information that would dramatically reduce the risks associated with this type of food is through reaching the minimal internal cooking temperature that these steaks have to be exposed to in order to sterilize the food and make it safe to eat. This information can be coupled with the labelling requirements suggested for meat processors or could be integrated as separate strategies.

To facilitate adaptation of these strategies, legislation or best practice guidelines by regulatory bodies would be an invaluable supportive tool in directing for these changes. Current attention and effort by regulatory bodies have mainly focused on controlling mechanically tenderized meats, which came as a result of realization that large outbreaks could be associated with the practice. Having had two years to decide on their position with mechanically tenderized meats, Health Canada and the Canadian Food Inspection Agency (CFIA) had recently voiced their response to the risk of MTBs. In February 2014, Health Canada issued a statement that they would be requiring proper labelling on MTBs which would clearly characterize it as mechanically tenderized products, and provide cooking instructions (Food and Drug Regulations, 2014). This would all be put into regulation so that the CFIA would be able to regulate labelling with meat processors and their products. A similar approach could be utilized for MTGase restructured meats, giving more authoritative power to responsible agencies to require meat processors to provide sufficient information to consumers on how to safely consume MTGase-restructured meats. As MTBs are in the transition to becoming more highly regulated, it is possible that other processing methods like MTBs will follow in the future by being as regulated. Otherwise, the only available guidance provided by PHAC and Health Canada for consumers is to continue to follow standard handling procedures which are too general to control improper consumption of MTGase restructured steaks (CPHA, 2012). Therefore directed guidance is required in 
order to provide the support needed to prevent the occurrence of foodborne illnesses with MTGase restructure steaks.

\section{Limitations}

One limitation to this study involves the technical aspect of creating MTGase-restructured steaks. The actual process could have been easier for individuals with culinary training or experience with this technique. The researcher in this case did not have the same experience, and notably created steaks that did not have a uniform distribution of effective bonds within the steak. Therefore these steaks may not truly represent the products that can be produced by professional processors, and may affect the observed results.

In addition, the size of steaks that were created, in retrospect, was not sufficiently big enough to accommodate the entire sensor region of the bimetallic thermometers. At times, the ends of thermometers were exposed to ambient temperature, which may have affected the final temperatures that were used to determine when to stop cooking.

Finally, a source of error could lie in the sample size chosen for this study. 30 samples, a minimal number of samples required, could not be attained, and only 26 samples were used for data analysis due to lack of resources to create more. A small sample size could always be a source of beta errors. Sample sizes were restricted by the cost of material and budget available.

\section{Recommendations}

As mentioned, one of the strategies that can be employed to help limit illnesses from MTGaserestructured steaks is to provide guidelines or supportive documents for all the regulators, processors, and consumers. This can help all groups become more knowledgeable on the subject, and enable consumers to limit their own exposure to undercooked restructured steaks. Currently, a risk assessment is being carried out on mechanically tenderized steaks by Health Canada, and a guideline could follow from the results (BCCDC, 2014). A similar guideline could be constructed for restructured steaks and other processing methods that threaten food safety.

Another way public health regulators can be involved in this situation is to make use of consumer advisories to help provide appropriate information to the public. Labels on publically available steaks will help educate the public who consume them at home. Alternatively, menu advisories, similar to the advisories in the United States for undercooked raw meat, would be useful for consumers to make an educated choice in restaurants and other food establishments to eat only fully cooked restructured meats (FDA, 2013).

\section{Future Research}

There are other techniques available in the food industry that can be tested for their capability of internalizing pathogens potential contribution to foodborne illnesses. A large family of products that is created through newly established processing techniques is known as functional foods (Zhang et al., 2010). Another example would be similar components to MTGase, which can bind meats, including whey and soy mixtures. These can be tested in the same manner as it has been done here for TGrestructured steaks.

As well, the Chancey study (2010) showed that application of lactic acid on mechanically tenderized steaks helped to limit the introduction of pathogens to the centre of these products. From a food technology perspective, this could be another aspect investigated to see if the same effect exists if steak components are treated with lactic acid before used for restructuring.

Finally, from a risk assessment perspective, it would be interesting to see how levels of E.coli could be reduced after being cooked to medium rare temperatures. If levels drop below a certain number (E.coli can cause illness with 100 cells), this would make exposure less of a problem.

As well, a second component that was suggested along with this study topic was to create a survey and assess the number of meat processors actually utilizing this technique and selling restructured 
steaks. More information on this topic can be acquired from BCCDC's Lorraine McIntyre.

\section{Conclusions}

Non-intact meats have frequently been seen as a contributor to foodborne illnesses in today's food supply. Items like steak, that have traditionally been considered as protected foods that don't require the same stringent compliance as non-intact meats with temperature control, are losing confidence from regulator and consumers due to the introduction of new processing methods. Transglutaminase restructured steaks are one such processed product that consumer groups are increasingly becoming of aware of. Through this study, it was shown that this type of meat can potentially harbor pathogens, and act as a vehicle for pathogens if heat treatment is not sufficient to inactivate them. Therefore, transglutaminase restructured steaks should not be consumed when cooked medium rare or less, otherwise it can be a significant cause of foodborne illness.

\section{Abbreviations}

TG: Transglutamainse

MTGase: microbial transglutaminase

MTB/MTM: mechanically tenderized beef/meat

E.coli: Escherichia coli

\section{Competing Interests}

The authors declare that they have no competing interests

\section{References :}

BCCDC. (2014). Meat. Retrieved from: http://www.bccdc.ca/foodhealth/meat/default.htm

BD. (2009). Difco \& BD Manual: Lauryl Tryptose Broth-Lauryl Sulfate Broth. Retrieved from: http://www.bd.com/ds/technicalCenter/misc/ difcobblmanual_2nded_lowres.pdf

Bottemiller, H. (2012). Meat industry defends 'meat glue' as safe, no secret. Food Safety News. Retrieved from: http://www.foodsafetynews.com/2012/05/m eat-industry-defends-meat-glue-as-safe-nosecret/\#.UmXZvShDsrU
Burton, J. (2012). Restructured chicken breast using activa RM - (aka transglutaminase or meat glue).

https://www.stellaculinary.com/podcasts/video/r econstructed-chicken-breast-using-activa-rmaka-transglutaminase-meat-glue

Canadian Public Health Association (CPHA). (2012). Information for Canadians on cooking mechanically tenderized beef. Retrieved from: http://www.cpha.ca/en/about/digest/xxxvi$\underline{3 / 10 . \operatorname{aspx}}$

Catford, A., Lavoie, MC., Smith, B., Buenaventura, E., Couture, H., Fazil, A., \& Farber, J. M.

(2013). Findings of the health risk assessment of Escherichia coli O157 in mechanically tenderized beef products in Canada.

International Food Risk Analysis Journal. 3. Retrieved from:

http://www.intechopen.com/journals/internationa l_food risk analysis journal/findings-of-thehealth-risk-assessment-of-escherichia-coli-o157in-mechanically-tenderized-beef-prod

Centers for Disease Control and Prevention. (2012). General Information Escherichia coli (E.coli). Retrieved from: http://www.cdc.gov/ecoli/general/index.html

Chancey, C. (2010). Survivability of E.coli O157:H7 in needle tenderized beef steaks during lactic acid application and cooking. [Dissertion] Retrieved from: http://repositories.tdl.org/ttuir/handle/2346/ETD-TTU-2010-12-1174

De Jong, G. A. H., \& Koppelman, S. J. (2002). Transglutaminase catalyzed reactions: Impact on food applications. Journal of Food Science. 67(8). Retrieved from: http://onlinelibrary.wiley.com/doi/10.1111/j.136 5-2621.2002.tb08819.x/abstract

Elizaquivel, P., Gabaldon, J. A., \& Aznar, R. (2011). Quantification of Salmonella spp., Listeria monocytogenes, and Escherichia Coli O157:H7 in non-spiked food products and evaluation of real-time PCR as a diagnostic tool in routine food analysis. Food Control, 22(2), 158-164. Retrieved from: http://www.sciencedirect.com/science/article/pii/ $\underline{\text { S0956713510001969 }}$

FDA. (2013). FDA food code 2009: Chapter 3-Food. Retrieved from: 
http://www.fda.gov/Food/GuidanceRegulation/R etailFoodProtection/FoodCode/ucm186451.htm\# part3-6

FDA. (2001). Transglutaminase: GRAS notification. Retrieved from: http://www.accessdata.fda.gov/scripts/fcn/gras_n otices/grn0095.pdf

Food network. (2013). Meat and poultry temperature guide. http://www.foodnetwork.com/recipesand-cooking/meat-and-poultry-temperatureguide/index.html

Hintze, J. (2013). NCSS 9. NCSS, LLC. Kaysville, Utah, USA. www.ncss.com.

Huang, L., \& Sheen, S. (2011). Quantitative analysis of vertical translocation and lateral crosscontamination of Escherichia coli O157:H7 during mechanical tenderization of beef. Journal of Food Safety. 31. Retrieved from: http://onlinelibrary.wiley.com/doi/10.1111/j.174 5-4565.2010.00273.x/abstract

Hygiena. (2013). Microsnap rapid microorganism detection. Retrieved from: http://www.hygiena.net/docs/MicroSnap_Brochu re_RevA042013_email.pdf

Motoki, M., \& Seguro, K. (1998). Transglutaminase and its use for food processing. Trends in Food Science \& Technology. 9(5). Retrieved from: http://www.sciencedirect.com/science/article/pii/ $\underline{\text { S0924224498000387 }}$

Microsoft. (2010). Excel.

Newell, D. G., Koopmans, M., Verhoel, L., Duizer, E., Aidara-Kane, A., Sprong, H., Osteegh, M., Langelaar, M., Threfall, J., Scheutz, F., Giessen, J., \& Kruse, H. (2010). Food-borne diseases-The challenges of 20 years ago still persist while new ones continue to emerge. International Journal of Food Microbiology, 139, S3-S5. Retrieved from:

http://www.sciencedirect.com/science/article/pii/ $\underline{\text { S0168160510000383 }}$

Public Health Agency of Canada. (2013). E.coli. Retrieved from: http://www.phac-aspc.gc.ca/fs$\underline{\text { sa/fs-fi/ecoli-eng.php }}$

Rattray, D. (nd). Meat temperature chart. Retrieved from: http://southernfood.about.com/library/info/blroas t.htm

Regulation amending the food and drug regulations (mechanically tenderizedbeef). (2014). Canada Gazette, Part I,February 14, 2014,148, No. 7.Retrieved from: http://www.gazette.gc.ca/rppr/p1/2014/2014-02-15/html/reg7-eng.php

Thomas, M. K., Murray, R., Flockhart, L., Pintar, K., Pollari, Fazil, A., . . .Marshall, B. (2013). Estimates of the burden of foodborne illness in Canada for 30 specified pathogens and unspecified agents, circa 2006. Foodborne Pathogens and Disease. 10(7). Retrieved from: http://online.liebertpub.com/doi/abs/10.1089 /fpd.2012.1389

Vrbova, L., Johson, K., Whitfield, Y., \& Middleton, D. (2012). A descriptive study of reportable gastrointestinal illnesses in Ontario, Canada, from 2007 to 2009. BMC Public Health. 12. Retrieved from: http://www.biomedcentral.com/1471$\underline{2458 / 12 / 970 /}$

Zhang, W., Xiao, S., Samaraweera, H., Lee, E. J., \& Ahn, D. U. (2010). Improving functional value of meat products. Meat Science, 86, 15-31 